\title{
Coulisses
}

Revue de théâtre

1 | Printemps 1990

Varia

\section{Barbey d'Aurevilly, le sagittaire critique de théâtre}

\section{Barbey d'Aurevilly}

Andrée Hirschi (éd.)

\section{CpenEdition}

\section{Journals}

Édition électronique

URL : http://journals.openedition.org/coulisses/1559

DOI : 10.4000/coulisses.1559

ISSN : 2546-9460

\section{Éditeur}

Presses universitaires de Franche-Comté

\section{Édition imprimée}

Date de publication : 1 avril 1990

Pagination : 49-53

ISSN : 1150-594X

\section{Référence électronique}

Barbey d'Aurevilly, « Barbey d'Aurevilly, le sagittaire critique de théâtre », Coulisses [En ligne], 1 | Printemps 1990, mis en ligne le 04 juillet 2017, consulté le 21 octobre 2019. URL : http://

journals.openedition.org/coulisses/1559; DOI : 10.4000/coulisses.1559

Ce document a été généré automatiquement le 21 octobre 2019

Coulisses 


\title{
Barbey d'Aurevilly, le sagittaire critique de théâtre
}

\author{
Barbey d'Aurevilly
}

Andrée Hirschi (éd.)

\section{NOTE DE L'ÉDITEUR}

Textes choisis et présentés par Andrée Hirschi.

Né à Saint-Sauveur-le-Vicomte (Manche), Jules Barbey d'Aurevilly s'installe à Paris ; il nourrit des ambitions politiques et rêve de devenir un homme d'action; ses tentatives dans le journalisme devraient l'y préparer. Cependant, de tempérament polémiste, il est incapable de se main- tenir dans la ligne d'un journal : toute carrière lui semble interdite. Par ailleurs, son œuvre romanesque - la plus connue étant Les Diaboliques n'a pas le succès escompté. À soixante ans, il a encore la position d'un débutant et contraint de se cantonner dans la critique théâtrale. Convaincu de l'infériorité du genre dramatique - exception faite des classiques - il n'accepte cette chronique que pour des nécessités purement matérielles. Entre 1866 et 1870, l'ensemble de ses articles forment les trois premiers volumes du Théâtre contemporain, qui en comporte cinq ${ }^{1}$. 


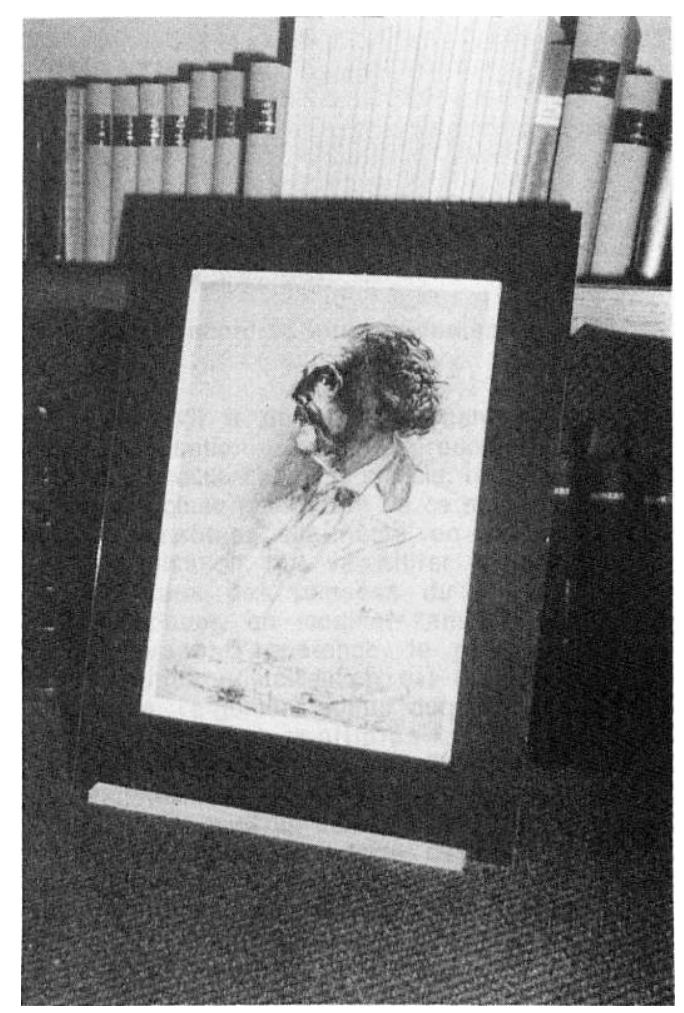

Vue de l'exposition Barbey d'Aurevilly.

2 À cette époque peu de pièces méritent qu'on s'y arrête :

Nous avons des amuseurs publics, - quand ils amusent, toutefois, - mais d'artistes dramatiques dignes de ce nom, cherchez cette aiguille d'or dans la botte de foin des sottises et des platitudes du théâtre actuel! Ceux qui ont été cette aiguille d'or ont perdu leur pointe... Ils se sont émoussés. M. Émile Augier, même pour ceux qui l'ont cru le plus fort du temps et qui l'appellent le grand Augier :

Lui disant tout Cyrus, dans leurs longs compliments, M. Émile Augier a vieilli et ses reprises valent mieux à présent que ses premières représentations. M. Sardou ne vit plus guère que sur son passé ; il ne convulsé plus les foules comme au temps de Patrie, ce convulsif, maintenant, à lui tout seul !

Quant à M. Alexandre Dumas, évidemment l'Octave de ce triumvirat, qui nous promet cependant une première représentation pour cet hiver, il se sent tellement au bout de son petit rouleau dramatique que, dans la préface d'une de ses dernières pièces, il nous a fait mélancoliquement ses adieux. Etait-ce là de la comédie encore?... ou, ce que je crois l'épuisement d'un esprit qui n'eût jamais ni l'abondance, ni le bouillonnement, ni le trop-plein. Voici les Trois Mousquetaires de la scène, mais ils en sont à Trente ans après !...

Seul, M. Gondinet, venu après eux, le petit Gondinet, vit encore ; mais M. Gondinet (singulier nom pour un Hercule!) aura-t-il les reins assez fermes pour soutenir les frises de ce théâtre que des cariatides fatiguées comme MM. Dumas, Augier et Sardou, menacent de laisser tomber? Sortirons-nous, grâce à lui de ce maigre régime forcé des reprises pour rentrer dans le régime plantureux des premières représentations?

(Th. IV, 140-141).

3 Pour Barbey, la littérature dramatique du XIX siècle lui apparaît réduite à quelques situations-types, que les auteurs se contentent d'exploiter :

$\mathrm{Au} \mathrm{XVIII}{ }^{\mathrm{e}}$ siècle, on ne cessa pas de comprendre une minute les grandes conditions essentielles de l'art dramatique. Diderot lui-même, ce satyre 
fumant d'un Matérialisme enivré, Diderot, l'auteur du Père de famille, ne les méconnut pas. Pour Diderot, comme pour les autres matérialistes d'un temps que nous voulons surpasser, le fait le plus pathétique ou le plus tragique ne produisait son effet à la scène que quand les préparations, des définitions approfondies avaient communiqué la vie morale aux personnages du drame et leur avaient créé une entité, une originalité, un caractère. Le caractère et la passion s'entrelaçant, se prenant à bras-le-corps dans une lutte superbe, tel était le but et les moyens du drame, quels qu'en fussent d'ailleurs les péripéties et le dénouement. On n'avait pas encore songé à substituer à ce jeu profond des passions et des caractères, kaléidoscope de l'âme humaine, de l'âme infinie, - l'intérêt brutal et stupide des situations, des portes ouvertes et fermées, les escarmouches de l'anecdote, et à remplacer les assises immortelles du drame par les chaussetrapes de l'imprévu. Pour cela, il fallait le XIX ${ }^{\mathrm{e}}$ siècle, et un ineffable matérialiste de sa façon! Ce matérialiste, inconscient de lui-même probablement, - car pour être conscient, il faudrait être réfléchi, et il l'était comme un vaudevilliste, - ne parait donc pas aussi majestueux que beaucoup d'autres matérialistes qu'on est en train de respecter. Mais je vous jure que les autres, qu'ils s'appellent comme ils voudront, n'ont pas certainement à leur manière le dixième d'influence que celui-ci exerce à la sienne! Nabot colossal, qui reste nabot quoiqu'il soit devenu un colosse, - un colosse comme il est un homme d'esprit, du reste, quoiqu'en art dramatique, en grand art, ce fût positivement un idiot, - cet idiot, le coryphée du siècle et des cabotins, a intronisé pour jamais peut-être sur la scène française des pantalonnades qui ont singulièrement favorisé et caressé toutes les paresses des spectateurs ignares et vulgaires. Des femmes surtout, des femmes en masse, et, pardessus le marché, celle des Directeurs de Théâtres, auxquels il est bien plus aisé de juger de l'effet d'un traquenard de situation que des analyses transcendantes du milieu moral et des caractères ; enfin, celle de l'acteur luimême, qui porte avec infiniment plus de légèreté les ficelles de l'imbroglio que les gigantesques replis de sentiments contradictoires qui s'enroulent et s'enfoncent dans les abîmes du cœur humain! Scribe a donc tout pris d'un seul coup de filet, non que son bras fût puissant ni que son filet fût vaste, mais parce qu'il péchait dans les bas-fonds de la sottise et de la lâcheté intellectuelles. Trouvé charmant par les crétins, la coqueluche de la Bourgeoisie, Scribe a créé une chose qui portera longtemps son nom, - le scribisme, - une pitié pour qui pense! mais, pour qui veut pénétrer au théâtre et s'y établir au nom d'un talent vigoureux et vrai, un obstacle. Parmi les impuissants qui ont fait du mal à quelque chose, il n'est personne qui en ait fait plus que lui à l'art dramatique, qu'il croyait bonnement son art... Personne non plus qui ait dépravé intellectuellement plus d'esprits sur une plus large surface. Guérira-t-on jamais de Scribe, de cet acarut dramatique ?... On peut en douter.

(Th. II, 73-74).

4 La moindre pièce est prétexte à mettre en cause l'art dramatique tout entier. Le premier feuilleton de Barbey est tout entier dirigé contre les directeurs de théâtre :

À prendre pour renseignement et pour guide l'histoire des Directions de Théâtre comme elle existe depuis plus de trente ans, et en supposant, ce qu'à Dieu ne plaise ! que cette histoire ne doive pas changer, les femmes à barbe ou sans barbe seraient aussi aptes à de telles besognes que les hommes qui, jusqu'ici, les ont faites. Elles n'y auraient certes pas montré, si on les y avait mises, plus d'incapacité radicale, d'ineptie naturelle, de petites passions, de vanité, de mépris des choses morales et littéraires que, sauf quelques exceptions bien rares, les hommes que nous y avons vus! Elles n'auraient pas fait reluire, dans ces charges d'âmes et d'esprits qu'on appelle des Directions de Théâtre, un écrin plus charmant et plus complet de tous 
les vices de gouvernement. Figurez-vous, en effet, qu'on l'écrivît, cette histoire! Figurez-vous qu'un Suétone de coulisses, - car il faudrait un, et qui écrivît en latin encore! - nous traçât l'histoire des Directeurs de Théâtre depuis environ trentecinq ans, de ces petits Césars et Césarions de derrière la scène dans lesquels pourrait manquer Néron, qui, le scélérat ! était un artiste, mais ne manqueraient ni Vitellius, ni Claude. Figurez-vous la chronique vraie et scandaleuse - et d'autant plus vraie qu'elle serait plus scandaleuse! - de ces grotesques et despotiques sultans Misapouf qui mènent leurs directions comme les sultans (les derniers!) menaient leur empire, quel monument plus curieux aurions- nous, et quelle explication meilleure de la corruption du Théatre et de son abaissement progressif jusqu'à ce profond degré d'abjection et de décadence où, présentement, nous le voyons? Mal- heureusement, ceux qui pourraient l'écrire l'ayant trop faite pour la raconter, il faut renoncer à cette histoire - lamentable et risible en même temps ! qui aurait les deux masques comme l'antique Génie de la Comédie elle-même : le masque qui rit et celui qui pleure, -celui-là du moins qui devrait pleurer ! Mais si nous ne l'avions pas et qu'il nous fallût regretter les annales détaillées de tout ce Bas-Empire, avec ses anecdotes, ses biographies, les secrets de ses Petits-Cabinets, toutes ces choses dont les rayons épars et concentrés font l'histoire, nous avons au moins ce qui suffit, aux moralistes comme aux hommes d'Etat, pour bien comprendre et bien juger. Nous avons les résultats obtenus, qui sont déplorables. Nous avons le Théâtre contemporain tout entier, qui se dresse comme une accusation et comme une lumière contre les Directeurs qui l'ont dirigé sur l'écueil, - l'écueil de vase où, en ce moment, il échoue !

(Th. I, 16-18)

Le second feuilleton s'ouvre sur une attaque virulente du Théâtre Français :

Le théâtre du Gymnase est présentement de fait, le premier Théâtre-Français. L'autre, qui en mérita longtemps le nom et qui le porte encore, n'est plus qu'une nécropole, - une chose morte et creuse, où reviennent des voix sépulcrales, quelque chose enfin comme l'Académie, cette institution de fantômes. J'en suis bien fâché pour les vieux classiques, mais c'est comme cela. Seulement, pour être plus vivant que ce grand bonhomme trépassé de Théâtre-Français, le Gymnase n'a pas pourtant de quoi en être bien fier...

(Th. I, 29)

6 Pour Barbey, il n'est pas question de raconter la pièce, comme tant d'autres critiques ; tout feuilletoniste se doit d'être gai ; sa critique est vive, amusante. Ainsi ajoute-t-il ce post-scriptum à un article sur une pièce qui l'avait ennuyé :

Ah! un incident. Un monsieur de l'orchestre est tombé sans connaissance au deuxième acte, et on l'a emporté. Comme cela il n'aura pas vu la pièce. C'est ma foi ! le plus spirituel d'entre nous.

(Th. XI, 235-236).

$7 \quad$ Il arrive parfois que Barbey blesse les vanités : l'actrice Duverger étant traitée avec trop d'indulgence par les autres critiques, Barbey se dé- chaîne, en affirmant qu'elle «ne serait jamais une comédienne et qu'elle n'est plus une jolie femme ». Outragée, $\mathrm{M}^{\text {lle }}$ Duverger le prit à partie un soir et menaça de lui lancer son éventail au visage. Barbey raconte l'incident :

Mademoiselle Duverger, que tout le monde connaît à Paris, et qui a joué, je crois, comme elle peut jouer, le rôle que tenait ce soir Madame Raucourt, était venue sans doute étudier, si elle est capable de le comprendre, comment on est une belle personne, très superbe en scène, autrement qu'avec des diamants, et comme on n'est pas une cabotine, mais une actrice. Le jeu, très applaudi, de Madame Raucourt, donnait sans doute beaucoup d'humeur à Mademoiselle Duverger ; mais moi, qui ai dit quelque part (je le reconnais humblement) qu'elle ne serait jamais une comédienne et qu'elle n'est plus une jolie femme, je lui en donnais bien davantage. 
Elle s'était déjà crispée en m'apercevant assez près d'elle, à l'orchestre ; mais lorsque ma lorgnette, qui, comme toutes les lorgnettes, faisait en mes mains le tour de la salle, se fut arrêtée un moment sur l'avant-scène où trônait cette douce et aima- ble personne, la voilà qui tout à coup se tord et m'apostrophe par mon nom, qu'elle dit en toutes lettres, ma foi ! me menaçant, si je la regardais encore, de me jeter son éventail à la tête. Quelle modération! Elle aurait pu me faire pis, en s'y jetant ; cela, du reste n'a pas été dit très haut, mais, dans le coin d'orchestre où nous étions, nous avons tous très bien entendu cette menace d'éventail, accompagnée de quelques autres arabesques et pantomimades expressives, et le rire nous a pris tous, et moi le premier, aux éclats de cette burlesque colère. J'étais étonné de l'aplomb de la demoiselle, à laquelle je croyais bien d'autres aplombs, mais pas celui-là. Mais étant, comme je fais profession de l'être, l'ennemi des fausses hérö̈nes, des amazones, des bas- bleus, des conférencières, et de toutes les hommasseries auxquelles les femmes maintenant prétendent, je n'étais pas fâché d'avoir contre toutes ces hommasseries un petit argument de plus. Elle me le donnait. Seulement, trouvant de mauvais ton toute scène publique (car, en particulier, je ne les hais pas), je ne dis point avec une femme, mais avec un porte-jupe quelconque, et ne voulant régaler la galerie d'aucun dialogue, je fis comme Beaumarchais, baissant les yeux devant Madame Gœzmann. Je me fis doux. Je ne fus point obstiné. Je ne fus pas taquin. Je fus bien gentil, comme disent les femmes, et je détournai ma lorgnette de ma Méduse aux yeux enflés et bien malades, hélas ! et qui me foudroyaient du fond de leur hydropisie. Cela me préserva-t-il ?... Toujours est-il que l'éventail annoncé, et que j'aurais renvoyé par l'ouvreuse, ne m'arriva point. Mademoiselle Duverger le garda, - et fit mieux que d'en cacher la petite honte de sa conduite; car elle s'en alla silencieusement bien avant la fin du spectacle, emmenée probablement par le vénérable personnage qui la mène partout, et qui prouva du moins qu'il était homme du monde en ne se mêlant point de cette ridicule incartade pour laquelle il l'aura peut-être grondée en rentrant... Nous, à qui elle n'a pas fait perdre notre bonne humeur, et dont, au contraire, elle a augmenté la gaieté, nous demandons qu'après tout on ne soit pas trop dur pour elle. L'impuissance de la colère d'un enfant en fait souvent toute la grâce, mais la colère est en soi un mouvement si bête qu'on peut la pardonner, quand même il n'y a là ni grâce, ni enfant !

Et d'ailleurs, franchement, si peu fat qu'on soit, ce n'est nullement désagréable de donner à une femme des sensations si vives... Malheureusement, nous n'aurons jamais à donner à Mademoiselle Duverger que celles-là.

(Th. II, 305-307) 


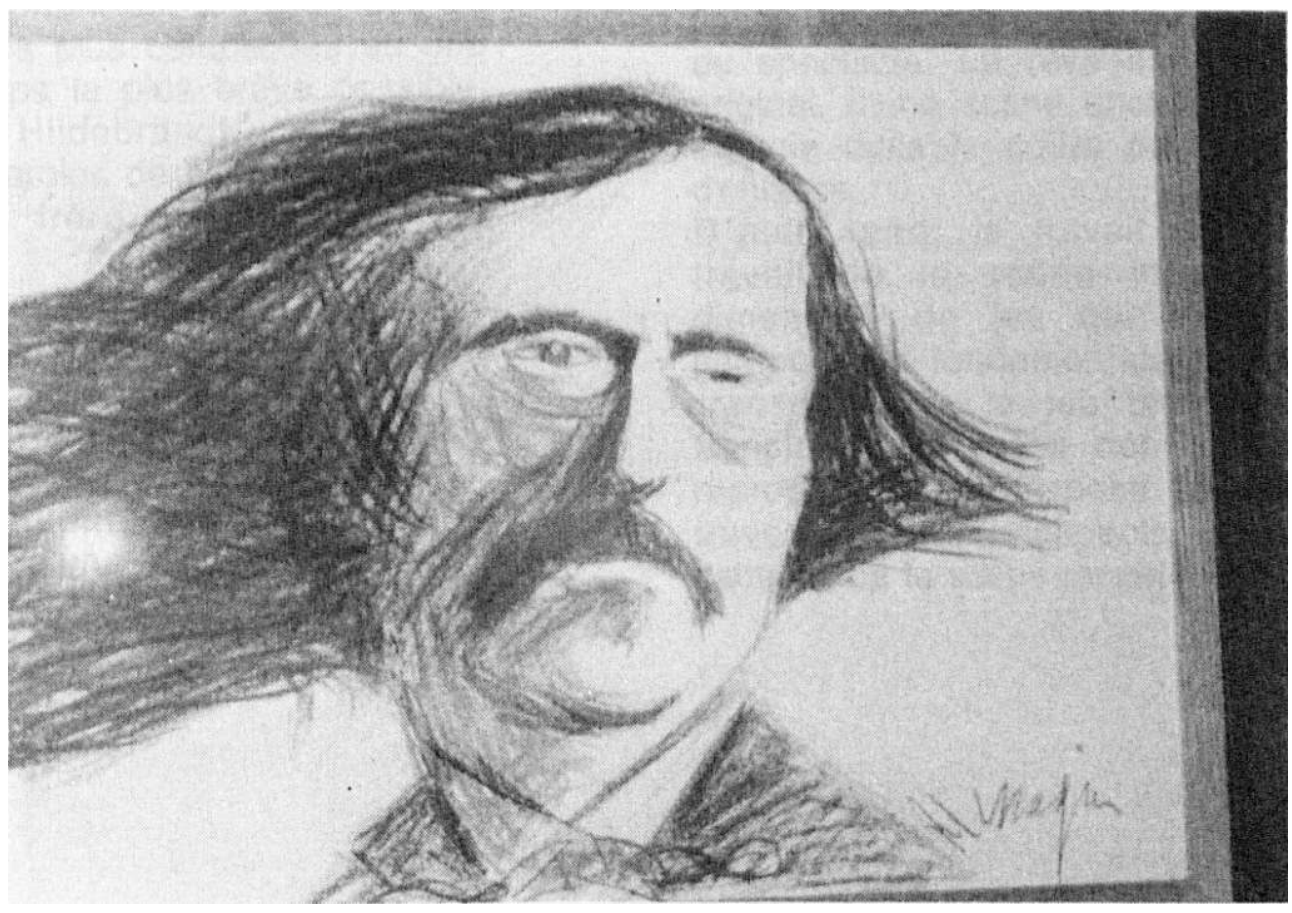

Portrait de Barbey par Messagier, avec l'aimable autorisation de l'auteur.

\section{NOTES}

1. Édités en 1887, 1988, 1989, 1992, 1996, ils sont actuellement introuvables. 